\title{
Acknowledgement of manuscript reviewers 2020
}

\author{
Florin Dumitru Mihaltan
}

\section{CONTRIBUTING REVIEWERS}

The editors of Tobacco Prevention \& Cessation would like to thank all our reviewers who have contributed to the journal in Volume 6 (2020).
AFFILIATION

1 Institute of Pneumology M. Nasta, Bucharest,

Romania

CORRESPONDENCE TO

Florin Dumitru Mihaltan. Institute of Pneumology

M. Nasta, Bucharest, Romania.

E-mail: editorial@tobaccopreventioncessation.com
Abasi, Hamid

Iran

Aissa, Khefacha

Tunisia

Alamer, Nora

United States

Alba, Luz-H.

Colombia

Allem, Jon-Patrick

United States

Alterman, Toni

United States

Alves, Elisabete

Portugal

Baezconde-Garbanati, Lourdes

United States

Basu, Saurav

India
Bernardes-Souza, Breno

Brazil

Bernert, John

United States

Bhuyan, Lipsa

India

Bialous, Stella

United States

Björk, Joar

Sweden

Bogdanovica, Ilze

United Kingdom

Bommelé, Jeroen

Netherlands

Bono, Rose

United States

Brandon, Thomas

United States
Branston, Robert J.

United Kingdom

Burdorf, Alex

Netherlands

Byron, Justin M.

United States

Callard, Cynthia

Canada

Cen Chen, Julia

United States

Chaaya, Monique

Lebanon

Chahar, Puneet

India

Chatterjee, Nilesh

India

Chaturvedi, Pankaj

India 
Chen, Yvonnes

United States

Chilkuri, Madhavi

Australia

Chinwong, Surarong

Thailand

Chowdhry, Aman

India

Clancy, Luke

Ireland

Collishaw, Neil

Canada

Correa Vazquez, Javier

France

Cosci, Fiammetta

Italy

Cruvinel, Erica

United States

Das, Smita

United States

Dauchy, Estelle

United States

Dautzenberg, Bertrand

France

Delahanty, Janine

United States

Delipalla, Sofia

Greece

Diamanti, Athina

Greece

Dotson, Jo Ann

United States
Dunbar, Michael

United States

Durgampudi, Praveen

India

East, Katherine

United Kingdom

Eddingsaas, Nathan

United States

Egbe, Catherine $\mathbf{O}$.

South Africa

Emery, Rebecca

United States

Ersel, Murat

Turkey

Feliu, Ariadna

Spain

Fernandes, Thiago

Brazil

Galimov, Artur

United States

Gendron, Fidji

Canada

Gérard, Audureau

France

Gettman, Lana

United States

Giannakopoulos, George

Greece

Godding, Veronique

Belgium

Gordon, Terry

United States
Gorini, Giuseppe

Italy

Gräsbeck, Helene

Finland

Gribben, Valerie

United States

Harri, Andrew

United States

Hart, Joy L.

United States

Hasegawa, Koji

Japan

Heyrani, Ali

Iran

Ho, Lai-Ming

China

Hollenbach, Jessica

United States

Hopkinson, Nick

United Kingdom

Hsu, Greta

United States

Hu, Yun

United States

Huber, Laurent

United States

Intarut, Nirun

Thailand

Jankowski, Mateusz

Poland

Jenson, Tara

United States 
Jia, Yuane

United States

Jia Hao, Jason See

Singapore

Jianhui, He

China

Jodalli, Praveen

India

Jorayeva, Anna

United States

Kashiwabara, Mina

Philippines

Kaufman, Pamela

Canada

Kava, Christine

United States

Kelly, Bryan

United States

Khalil, Georges

United States

Kilibarda, Biljana

Serbia

Kollath-Cattano, Christy

United States

Králíková, Eva

Czech Republic

Krasovsky, Konstantin

Ukraine

Kyriakos, Christina

Belgium

Lam, Tai Hing

Hong Kong
Laverty, Anthony

United Kingdom

Li, Xiaoyin

United States

Liao, Yanhui

China

Liber, Alex

United States

Lim, Kuang Hock

Malaysia

Lisha, Nadra

United States

Livingstone-Banks, Jonathan

United Kingdom

Majumdar, Anindo

India

Mamudu, Hadii

United States

Mantey, Dale

United States

Marshall, Anna-Marie

United Kingdom

Martinez, Ursula

United States

Marzo, Roy

Malaysia

McMillen, Robert

United States

Mehta, Vini

India

Mentis, Alexios

Greece
Mercincavage, Melissa

United States

Minaker, Leia

Canada

Mold, Alex

United Kingdom

Myers, Mark

United States

Nandi, Arindam

United States

Neuner, Bruno

Germany

Nicholson, Jody

United States

Niemeier, Brandi

United States

Nikitara, Katerina

Greece

Nohavova, Iveta

Czech Republic

Obeidat, Nour

Jordan

Odani, Satomi

Greece

Oliver, Jason

United States

Olowookere, Samuel

Nigeria

Ortiz-Chacha, Christian

Mexico

Ortiz-León, María Cristina

Mexico 
Osibogun, Olatokunbo

United States

Özmen, Utku

Turkey

Pacifici, Roberta

Italy

Pappas, Steven

United States

Park, Myung-Bae

Korea

Parks, Michael

United States

Pawar, Sudarshana

India

Pénzes, Melinda

Hungary

Peruga, Armando

Chile

Pignataro, Rose

United States

Precioso, José

Portugal

Ridlo, Ilham

Indonesia

Russo, Patrizia

Italy

Saatci, Esra

Turkey

Saliba, Najat

Lebanon

Samara, Athina

Greece
Sarode, Sachin

India

Saygın, Avşar Tuba

United Kingdom

Sebayang, Susy

Indonesia

Sharan, Rajeshwar

India

Sharma, Surendra

India

Shiffman, Saul

United States

Shinaba, Muftau

United States

Shishani, Kawkab

United States

Siddiqi, Umar

United States

Snyder, Marsha

United States

Son, Youn-Suk

Korea

Song, Fujian

United Kingdom

Soulakova, Julia

United States

Stillman, Frances

United States

Stoklosa, Michal

United States

Stuppy Romeo, Kelsey

United States
Surapaneni, Krishna Mohan

India

Tadesse, Tamiru

Ethiopia

Talhout, Reinskje

Netherlands

Tami-Maury, Irene

United States

Tateno, Hiroki

Japan

Thirlway, Frances

United Kingdom

Thomas, Dennis

Australia

Thomas, Roger

Canada

Timberlake, David

United States

Trofor, Antigona

Romania

Tseng, Tung-Sung

United States

Tzortzi, Anna

Greece

van Walbeek, Corne

South Africa

Vitória, Paulo

Portugal

Wadgave, Umesh

India

Wamamili, Ben

New Zealand 
Watts, Christina

Australia

Willand, Nicola

Australia

Yang, Yong

United States
Zeebari, Zangin

Sweden

Zeskind, Philip

United States

Zhou, Li

China

\section{Zisis, Konstantinos}

Greece 